\section{Implementation of Palliative Care in Palestine: Cultural and Religious Perspectives}

\author{
Rania Abu Seir, PhD; Akram Kharroubi, PhD \\ Department of Medical Laboratory Sciences, Faculty of Health Professions, Al-Quds University, \\ Abu Dies, Palestine
}

\section{INTRODUCTION}

The care of terminally ill cancer patients poses a significant global public health problem. ${ }^{1}$ Populations are growing older as healthcare interventions become increasingly more effective in the management of chronic diseases. ${ }^{2}$ Care of the terminally ill patients has evolved significantly over the centuries in the developed countries from Hospice services to highly specialized palliative care programs and facilities. In the Middle East, the progress in this area has been very slow over the last two decades which could be attributed to several reasons including the lack of education, training, budgets and several other barriers. ${ }^{3}$ Although age projection for Palestinians does not indicate generational transition toward an older population, ${ }^{4}$ yet the increasing incidence of cancer cases and other chronic diseases that need palliation, alerts the policy makers to the crucial need of introducing and developing palliative care services. Thus, the aim of this paper is to: 1) Reflect on the need and access of the Palestinians living in the West Bank and Gaza Strip to palliative care; 2) Define the factors that might interfere with the proper introduction of palliative care; and 3) Focus on the positive compensatory effect of religion and culture on palliative care.

The Palestinian population in the West Bank and Gaza Strip at the end of 2016 was 4.88 million $\left(61 \%\right.$ and $39 \%$, respectively. ${ }^{4}$ Palestinians live in refugee camps were about $41.9 \%$ distributed between the West Bank and Gaza strip, and $16.7 \%$ live in rural areas $(2.7 \%$ in Gaza Strip compared to $25.6 \%$ in the West Bank. ${ }^{4}$ The Palestinian population is a young generation, though it has been gradually aging; birth, fertility, and death rates have been dropping. In fact, the median age of the Palestinians was 16.4 years in 2000 and increased to 20 years in 2016, with $39.1 \%$ of the population under 15 years old compared to $2.9 \%$ over 65 years of age. The life expectancy of the population was 73.7 years in 2016 compared to 72.4 years in $2011 .^{4,5}$ The Palestinian population has low income, and the majority do not have health insurance and cannot afford to seek medical care. ${ }^{6}$

\section{Palliative Care Definition}

A wide range of brief and comprehensive definitions of palliative care have been published. World Health Organization (WHO) defined palliative care as "an approach that improves the quality of life of patients and their families". Three palliative care forms exist according to the need of the patient: palliative approach, specialized palliative services and end-of-life/terminal care. A palliative approach focuses on the improvement of the quality of life (QoL) for terminally ill patients and their families; specialized palliative care supports the initial palliative plan through a specialist individual or a team to provide advice and interventions; and the end-of-life (terminal) care focuses on patients in their final days or weeks of life including the patient's physical, emotional and spiritual comfort through medication management of symptoms and support for the patient's family. ${ }^{8}$ 


\section{Palestinian Palliative Care Needs in Perspective}

Although palliative care focuses on cancer patients, it recognizes and targets all groups experiencing serious pain, including patients with cardiovascular diseases (CVDs), chronic respiratory diseases, HIV, dementia, and diabetes. ${ }^{9,10}$ There are several approaches used to estimate the size of palliative care needs. One approach is to estimate $75 \%$ of deaths; another one relies on mortality rates for specific diseases like cancer, CVDs, diabetes, HIV, dementia, senility, renal diseases, and chronic respiratory diseases. ${ }^{11}$ In the Palestinian community, CVDs were reported as the leading cause of death in 2015 with 3,484 deaths $(27.5 \%$ of all deaths). ${ }^{6}$ Moreover, cerebrovascular diseases account for $9.9 \%$ of all deaths. ${ }^{6}$ As for cancer, it has become the second leading cause of death since 2011 comprising $13.8 \%$ of all deaths of according to the last Ministry of Health (MOH) report in 2015. Mortality rates were 41.9 and 36 deaths per 100,000 people in the West Bank and Gaza Strip, respectively. $6,10,12$ As projected by the WHO, estimates of death in 2030 for CVDs world-wide and in Eastern Mediterranean Region were almost 24 million and 2.1 million compared to 19.4 million and 1.44 million in 2015 , respectively. For cancer, world-wide deaths are estimated to be 12 million in 2030 (17.6\% from worldwide expected deaths). ${ }^{13}$ In Eastern Mediterranean Region, 630,000 deaths are projected to occur in 2030 compared to 402,000 deaths reported in $2015 .{ }^{13}$

Globally, it is estimated that 20 million patients or more need palliative care at the end-of-life and this number doubles if taking into consideration those patients at earlier stages of illness that could also benefit from such care. ${ }^{1}$ Nevertheless, only 3 million end-of-life patients receive palliative care, and although most global palliative services are provided in developed countries, $80 \%$ of needs are required by low- and middle-income countries. ${ }^{1}$ Moreover, late diagnosis of cancer poses a significant problem in the Middle East. Most cases (68\%) are diagnosed in stages III and IV of the disease when the individual is terminally ill, needs aggressive treatment strategies and have limited chances of survival. ${ }^{14}$ In Saudi Arabia and Egypt, more than 70\% of breast cancer cases are diagnosed at advanced stages with or without metastasis. ${ }^{15}$ Upon assessment of QoL of cancer patients in the West Bank, poor scores in all QoL domains were revealed. Poorer QoL functioning was associated with sex, income, pain, fatigue and stage of cancer. ${ }^{14}$ In the Eastern Mediterranean Region, only $5 \%$ of patients who need palliative care receive it. ${ }^{15}$

\section{Palestinian Palliative Care Facilities, Education, and Research}

Despite the development in the advancements Palestinians have witnessed in the last few years, cancer care is still suffering from major deficiencies. Only seven hospitals provide cancer care for the Palestinians; two governmental hospitals located in the West Bank (Beit Jala and Al-Watani), and two non-governmental facilities (Augusta Victoria and An-Najah National University Hospitals). The other three hospitals are governmental organizations located in Gaza Strip (Al-Shifa, European, and Al-Rantisi Specialized Pediatric Hospitals). Palliative care services are not provided in any of these hospitals, and specialized palliative care doctors or nurses are completely lacking.

The only registered non-governmental organization (NGO), Al-Sadeel Society, provides palliative care services in Bethlehem focusing on educating physicians, nurses, patients and their families about fundamental practices. The organization has two qualified palliative care workers (a nurse and a social worker). This NGO provides consultation for cancer patients and their families through its team along with occasional consultations from a trained oncologist who received six months of training in palliative care. ${ }^{16}$ Recently, Al-Sadeel started a recent program with the $\mathrm{MOH}$ and Augusta Victoria Hospital at IbnSina College for Health Sciences to train undergraduate nursing students on palliative care. ${ }^{12,16}$ Hospice and end-of-life care services are not available for terminally ill Palestinian patients, and the teams working with cancer patients and geriatric departments lack knowledge and training in these areas. ${ }^{16}$ In the West Bank and Gaza, palliative care is not a requirement in medical and nursing curricula and training programs. Although, recently Bethlehem University in the Southern West Bank started a higher diploma program in oncology nursing.

Availability of research in a particular country reflects the size of attention directed toward developing palliative care. Few researches were conducted in West Bank and Gaza Strip to assess this need. ${ }^{16,17}$ A recent, small-scale study conducted in Gaza Strip reported low attitude toward caring for dying patients among nursing students at the Islamic University of Gaza, which was attributed to lack of knowledge and skills. ${ }^{17}$ Another crosssectional study assessed the knowledge and attitude of nurses towards palliative care in the West Bank. The study included 120 nurses working in the three hospitals located in the Northern region, $45.8 \%$ of the subjects had a low-level of knowledge in palliative care, whereas $56.2 \%$ had a moderate score for attitude towards palliative care. The study also found a significant influence of the qualifications, years of experience and palliative care training, on knowledge scores among Palestinian nurses. ${ }^{18}$

\section{Pain Assessment and Management}

In terminally ill patients, pain is one of the most commonly experienced symptoms. Worldwide, about $80 \%$ of cancer patients and more than $60 \%$ of patients with advanced CVDs reported moderate to severe pains. ${ }^{9}$ Pain assessment and frequent reassessment using proper instruments with reported psychometrics is crucial, ${ }^{19}$ and the most comprehensive tools are the multidimensional ones. To achieve optimal treatment, pain intensity, severity, location, timing, relieving factors and interference with the daily activities should be essential components of a pain assessment tool. In developing countries like the Middle East, insufficient knowledge of healthcare providers on pain assessment and treatment, high cost and limited resources, strict policies and regulations regarding prescription of narcotic drugs contribute to the low utilization of palliative care pharmacological agents..$^{20,21}$ 
In 1986, the WHO developed global strategies to control cancer pain and the cornerstone was the permanent availability of opioid analgesics. Opioids are the most efficient narcotic drugs used for the relief of moderate to severe pain in cancer patients; examples are morphine, oxycodone, and fentanyl. Furthermore, as pain is documented to be associated with depression and anxiety, benzodiazepine anxiolytics and other psychotropic drugs have been used to treat neuroglial and mental cancer-related disorders. ${ }^{22}$

In the developing countries, pain medications are still scarce. The WHO and the International Narcotics Control Board (INCB) use consumption of morphine as an indicator of access to palliative care. ${ }^{21}$ Despite the unavailability of statistics and accurate information on narcotics' consumption in the West Bank and Gaza, some crude information from the Palestinian $\mathrm{MOH}$ showed the distribution of consumption of narcotic drugs and psychotropic substances in governmental and private health sectors in $2015 .{ }^{6}$ The reported data reflected the amounts of narcotic use including pethidine, fentanyl, and morphine in their different concentrations and forms. However, the reported values did not differentiate between the use for the purpose of palliative care of terminally ill patients or post operation pain relief. ${ }^{6}$ The INCB reported the average consumption of narcotic drugs in several Middle Eastern countries between 2007-2009, but Palestine was not included. ${ }^{22}$ Fadhil and her colleagues reported a small level of opioids consumption ( $0.384 \mathrm{mg}$ per person) in the Eastern Mediterranean Region compared to the rest of the world (6.24 mg per person). ${ }^{15}$ Regarding prescription of medications and especially pain medications and opioid drugs, Palestinian nurses have no privilege in ordering or modifying medications. ${ }^{16}$

Religious and Cultural Aspects: Islamic Perspective on Illness, Pain Management, Palliative Care and Euthanasia

Religious aspects: The majority of the Palestinians living in the West Bank and Gaza are Muslims, with less than $2 \%$ Christians. Religion and spirituality are related parts of the human personality, and a cornerstone of palliative care; they affect the way in which patients cope with their illness, and spiritual care is as important to the families as to the patients themselves. ${ }^{23}$ Furthermore, they help the patients to find "inner strengths" and to "think positive" which consequently empower them to deal with the suffering resulting from their terminal illness. ${ }^{24}$

Muslims believe in Allah (God) and in the inevitability of death, and that death and illness are God's will that cannot be avoided or stopped. Eternity is in the hereafter and whatever we do in this temporary life on earth will be either rewarded or punished for in the hereafter. This belief helps patients to cope with illness and die in peace looking forward to the reward in the hereafter. Furthermore, from an Islamic perspective, illness and suffering are accepted as a way of atonement of sins. It brings a Muslim closer to God, makes him more thankful and strengthens his belief. Also, it is considered as an evidence of accepting God's will, a reminder of the weakness of a human being and a sign of how close death could be. It is received as a test from God for the strength of faith as prophets being the most tested "Indeed, those who patiently persevere will truly receive a reward without measure" (Zumar 39.10), and a reminder of the bounties of God such as the bounty of health. All of this does not conflict with the necessity of giving all efforts to relief suffering. ${ }^{25}$ According to most Muslim scholars, seeking treatment for illness is permissible, and the duty of pursuing treatment increases with increasing the seriousness of the disease. Otherwise, the patient will commit a sin if he does not pursue treatment for serious illness. ${ }^{25,26}$ However, using opioids as pain killers to reduce the level of pain, and not as a medication, is permissible, but not mandatory. On the contrary, if a Muslim decides to tolerate the pain and not take pain killers, he will be rewarded more in the hereafter.

Euthanasia: According to Islamic teachings, the person is entrusted on his life, and it is not his choice or the choice of the family (passive euthanasia) or the doctor to end it (active euthanasia), but to protect it. Islam prohibits euthanasia or killing a patient as a mean of mercy. Allah (God) says in the Qur'an:

\section{“... and do not cast yourselves into destruction with your own hands" (Al-Baqra 2:195) \\ “... and do not (commit suicide) kill yourselves [or one anoth- er]. Indeed, Allah is most Merciful to you” (An-Nisaa 4:29)}

Islam encourages seeking medications and doctors should look for all means and work hard to treat the patient and make his life comfortable. Doctors should not listen to patients if they request their life to be ended even if it is a rational valid refusal (passive euthanasia). This is also in accordance with the Hippocratic Oath for medical doctors "I will not give a lethal drug to anyone if I am asked, nor will I advise such a plan", ${ }^{27}$ The doctor is considered a killer in Islam and will be punished as such if he applies euthanasia to any patient. In the hereafter, God also punishes those who commit suicide even if the reason is the intolerable pain. Most Muslim scholars consider the following cases as not being euthanasia (active or passive): first, when stopping medication by the supervising medical committee if proven ineffective including ending life supporting machines or any other interventions like resuscitation for patients diagnosed as "brain dead;" second, when the patient refuses to administer a medication that the medical committee firmly believes that it won't be effective; and third, increasing medication doses to reduce pain unless the medical committee is confident that increasing it will kill the patient. ${ }^{28}$

Like Islam, the teachings of Christianity also prohibit euthanasia, and the culture of both Palestinian Muslims and Christians is nearly the same in respecting life as a gift from God, and suicide and euthanasia are considered sins and not practiced by the Palestinian society.

Cultural aspects: Palestinian set of cultural values are mainly derived from religion. Culture is an important determinant in 
healthcare because of its influence on lifestyle, beliefs and values, the perception of individuals' QoL and palliative care, the interaction with healthcare system and decision making throughout illness and end-of-life. ${ }^{29}$

Despite the undergoing changes in the social structure, the Palestinian family has an extended structure rather than nuclear. Children live with their parents until they are old enough to get married and build their families, and they maintain proximity to their parent even after marriage. Family members gather frequently and in different social and religious occasions. The respect for elderly and filial piety are integral parts of the social and religious values of the community. Similarly, other cultural values include the care and help to the weak and ill; decision making is collective and involves parents, spouses and older members (brothers, sisters, grandparents, etc); and family and familial bonds are sacred. Palestinian Christian families have the same values of extended families and respect for the elderly as Palestinian Muslims.

Spouses, friends, and neighbors stand by each other's side; it is the family's duty to take care of the ill members, to be there for them in weakness and pain, at the end-of-life and the moments of death. What constitute a "good death" in this population for the ill members are dying surrounded with family and at home and securing their families before they leave. ${ }^{30}$ When someone is terminally ill, family members in most cases prefer to take the patient home to be around him to provide comfort and company, friends and relatives tend to go and visit the patient and stay with him for a while. One of the common practices of Muslims is reading verses from the Holy Qur'an for patients which might be a comfort. ${ }^{3}$

Among Arabs, the desire of appearing strong and to please others at all costs, bearing physical pains, hiding emotions, staying at the head of responsibility, performing duties and playing roles without admitting the need for help or showing signs of weakness are important factors in maintaining one's dignity. ${ }^{31}$ On the other hand, this factor may contribute to the high rates of late diagnosis and refusal of palliative care, and pain treatments and psychological interventions. ${ }^{32,33}$

\section{Barriers to Palliative Care}

The Palestinians living in the West Bank and Gaza suffer from the same barriers reported by the neighboring Arab countries including the lack of support by health policies and education, little or no knowledge of the principles and practices of palliative care among healthcare providers and patients and their families. In addition to the weak healthcare system and the scarcity of resources and unavailability of medications especially opioids, ${ }^{1,15,22}$ the Palestinians have additional barriers due to the peculiar political situation since the West Bank and Gaza governed by the Palestinian authority (PA) are still under Israeli occupation.
Despite the lack of resources, the PA provides full coverage for cancer patients' diagnosis and treatment and to many people injured by wars and conflicts. In Gaza, more than 18,000 persons were injured because of three wars in the last ten years. Most of the injured require medications and in most cases opioids. This burden can't be tolerated by the PA government whose budget was about four billion U.S. dollars in 2016 and only $8.5 \%$ was allocated to health. Around $32 \%$ of the health allocated budget is usually used to cover referrals to Israeli and neighborhood hospitals due to the shortage or lack of advanced diagnostic tests and treatment for cancer and other diseases in the West Bank and Gaza. Thus, an additional budget should be allocated to improve the Palestinian Healthcare System (PHS). Moreover, tremendous efforts and support to the PHS are required to introduce palliative care at the level of training and education of the healthcare providers and the patients and their families. Further, financial support is required to the PA to implement a national palliative care policy including "a ready access of suffering patients to opioids" according to the recommendations of the WHO in 2002. ${ }^{34}$

\section{Interventions and Recommendations}

Introducing and implementing palliative care for Palestinians in the West Bank and Gaza require integrated local and international efforts and support. At the local level, Palestinian ministries and stakeholders should place palliative care on their priority list. Additional budget should be allocated to the $\mathrm{MOH}$ to capacity build the human resources and the premises of the Palestinian healthcare system. The extra budget should be invested in improving cancer screening, early and proper diagnosis, and follow-up, which consequently will decrease the number of terminally ill patients who are in need of palliative care. Moreover, professional and well-structured training programs should be conducted especially in hospitals with oncology and geriatric departments to improve the qualifications of the healthcare providers and to train them to adopt the skills and the attitudes that are part of palliative care. The international community such as WHO, Middle East Cancer Consortium (MECC) and other cancer-related organizations could significantly contribute to this training. Furthermore, a national policy and legislation regarding palliative care provided at hospitals and homes should be implemented and integrated with the healthcare system and the health insurance to warrant the availability and access to opioids.

The Palestinian Ministry of Education and Higher Education should also take the initiative to integrate palliative care in the medical and the nursing curricula and encourage them to develop specialized tracks in this field. Master programs should also be offered by the Palestinian universities to improve the qualification of the healthcare professionals and to motivate research conduction in palliative care.

Finally, the Palestinian culture that stems mainly from religion compensates with increased psychological comfort and spirituality, especially for terminally ill patients. Improving pal- 
liative care among Palestinians requires not only to implement the above interventions but also to strengthen the positive religious and cultural values in caring for the sick people particularly with the new generation where these values tend to weaken with time.

\section{CONFLICTS OF INTEREST}

The authors declare that they have no conflicts of interest.

\section{REFERENCES}

1. Worldwide Palliative Care Alliance (WPCA) and World Health Organization (WHO). Global atlas of palliative care at the end-of-life. 2014. Web site. http://www.who.int/nmh/Global_Atlas_of_Palliative_Care.pdf. Accessed March 22, 2017.

2. Stjernsward J, Clark D. Palliative medicine-a global perspective. In: Doyle D, Hanks G, Cherny N, Calman K. eds. Oxford Textbook of Palliative Medicine. $3^{\text {rd }}$ ed. Oxford, USA: Oxford University Press; 2003: 1197-1224.

3. Aljawi D, Harford J. Palliative care in the Muslim-Majority Countries: The need for more and better care. In: Chang E, Johnson A. eds. Contemporary and Innovative Practice in Palliative Care. China: InTech; 2012: 137-150.

4. Palestinian Central Bureau of Statistics (PCBS). Palestinians at the End of 2016. 2016. Web site. http://www.pcbs.gov.ps/ Downloads/book2242.pdf. Accessed March 22, 2017.

5. Palestinian Central Bureau of Statistics (PCBS). Palestinians at the end of year 2011. 2011. Web site. http://www.pcbs.gov.ps/ Downloads/book1815.pdf. Accessed March 22, 2017.

6. Palestinian Ministry of Health (MOH). Health Annual Report, Palestine. 2015. Web site. http://moh.ps/Content/Books/ NWNJXX7RJ92Bn4f5EGYiH43a2tjAAzKBnseGnEUCaqWqYZndsbCcPy_JQWguvkHTR4Xk4zUpdT45ooWxH11BhIbVAxwpGWy2wiwHdGcM5K7aZ.pdf. Accessed March $22,2017$.

7. World Health Organization (WHO). WHO Definition of Palliative Care. 2017. Web site. http://www.who.int/cancer/palliative/definition/en/. Accessed March 22, 2017.

8. Brisbane South Palliative Care Collaborative (BSPCC). Guide to the Pharmacological Management of End-of-life (Terminal) Symptoms in Residential Aged Care Residents. 2013. Web site. https://www.caresearch.com.au/Caresearch/Portals/0/PA-Tookit/Guide\%20_to_the_Pharmacological_Management_of_End of_Life(Terminal)Symptoms_in_Residential_Aged_Care_Residents_1.pdf. Accessed March 22, 2017.

9. World Health Organization (WHO). Palliative Care. 2015. Web site. http://www.who.int/mediacentre/factsheets/fs402/en/.
Accessed March 22, 2017.

10. World Health Organization (WHO). Cancer control: Knowledge into action: WHO guide for effective programmes; module 5. 2007. Web site. http://apps.who.int/iris/bitstream/10665/44024/1/9241547345_eng.pdf. Accessed March $22,2017$.

11. Murtagh FE, Bausewein C, Verne J, Groeneveld EI, Kaloki YE, Higginson IJ. How many people need palliative care? A study developing and comparing methods for population-based estimates. Palliat Med. 2014; 28(1): 49-58. Web site. http:// journals.sagepub.com/doi/abs/10.1177/0269216313489367. Accessed March 22, 2017.

12. Kharroubi AT, Abu Seir RY. Cancer Care in Palestine. In: Silbermann M. ed. Cancer Care in Countries and Societies in Transition: Individualized Care in Focus. New York, USA: Springer International Publishing; 2016: 77-97.

13. World Health Organization (WHO). Projections of mortality and burden of disease, 2004-2030. 2015. Web site. http://www. who.int/healthinfo/global_burden_disease/projections2004/en/. Accessed March 14, 2017.

14. Shawawra M, Khleif A. Palliative care situation in palestinian authority. J Pediatr Hematol Oncol. 2011; 33(Suppl 1): S64S67. doi: 10.1097/MPH.0b013e31821223a3

15. Fadhil I, Lyons G, Payne S. Barriers to, and opportunities for, palliative care development in the eastern mediterranean region. Lancet Oncol. 2017; 18(3): e176-e184. doi: 10.1016/ S1470-2045(17)30101-8

16. Khleif M, Dweib A. Palliative Care and Nursing in Palestine, 2015. Journal of Palliative Care and Medicine. 2015 (S4:003).

17. Abu-El-Noor NI, Abu-El-Noor MK. Attitude of palestinian nursing students toward caring for dying patients: A call for change in health education policy. J Holist Nurs. 2016; 34(2): 193-199. Web site. http://journals.sagepub.com/doi/ abs/10.1177/0898010115596492. Accessed March 22, 2017.

18. Ayed A, Sayej S, Harazneh L, Fashafsheh I, Eqtait F. The nurses' knowledge and attitudes towards the palliative care. J Edu Pract. 2015; 6(4): 91-99. Web site. https://eric. ed.gov/?id=EJ1083747. Accessed March 22, 2017.

19. Vincent Perron M, Ronald S. Schonwetter M, FACP. Assessment and management of pain in palliative care patients. Cancer Control. 2001; 8(1): 15-24.

20. Caroll P. Palliative Care in the Middle East. ASCO Connection. 2014. Web site. https:/connection.asco.org/magazine/ features/palliative-care-middle-east. Accessed March 22, 2017. 
21. Bruera E, Lima Ld, Wenk R, Farr W. Palliative Care in the Developing World: Principles and Practice. Houston, TX, USA: IAHPC Press; 2004.

22. Silbermann M, Arnaout M, Daher M, et al. Palliative cancer care in middle eastern countries: Accomplishments and challenges. Ann Oncol. 2012; 23(Suppl 3): 15-28. doi: 10.1093/annonc/mds084

23. Delgado-Guay MO. Spirituality and religiosity in supportive and palliative care. Curr Opin Support Palliat Care. 2014; 8(3): 308-313. doi: 10.1097/SPC.0000000000000079

24. Lopez-Sierra HE, Rodriguez-Sanchez J. The supportive roles of religion and spirituality in end-of-life and palliative care of patients with cancer in a culturally diverse context: A literature review. Curr Opin Support Palliat Care. 2015; 9(1): 87-95. doi: 10.1097/SPC.0000000000000119

25. Bushnaq M. Palliative care in Jordan: Culturally sensitive practice. J Palliat Med. 2008; 11(10): 1292-1293. doi: 10.1089/ jpm.2008.0166

26. Padela AI, Qureshi O. Islamic perspectives on clinical intervention near the end-of-life: We can but must we? Med Healthcare Philos. 2016. doi: 10.1007/s11019-016-9729-y

27. Barns JW. The hipocratic oath. An early text. $\mathrm{Br}$ Med $J$. 1964; 2(5408): 567.

28. Ayuba MA. Euthanasis: A muslim's perspective. Scriptura.
2016; 115(1): 1-13. doi: 10.7833/115-0-1175

29. Saca-Hazboun H, Glennon CA. Cultural influences on healthcare in Palestine. Clin J Oncol Nurs. 2011; 15(3): 281. doi: 10.1188/11.CJON.281-286

30. Tayeb MA, Al-Zamel E, Fareed MM, Abouellail HA. A "good death": Perspectives of muslim patients and healthcare providers. Ann Saudi Med. 2010; 30(3): 215-221. doi: 10.4103/02564947.62836

31. Cohen M. Multicultural aspects of care for cancer patients in Israel. In: Surbone A, Matjaž Zwitter, Rajer M, Stiefel R, eds. New Challenges in Communication with Cancer Patients. $1^{\text {st }}$ ed. New York, USA: Springer; 2012: 317-331.

32. Lipson JG, Meleis AI. Issues in healthcare of middle eastern patients. West J Med. 1983; 139(6): 854-861.

33. Farhat F, Othman A, El Baba G, Kattan J. Revealing a cancer diagnosis to patients: Attitudes of patients, families, friends, nurses, and physicians in Lebanon-results of a cross-sectional study. Curr Oncol. 2015; 22(4): e264-e272. doi: 10.3747/ co.22.2351

34. World Health Organization (WHO). National cancer control programmes: Policies and managerial guidelines. 2002. Web site. http://www.who.int/cancer/nccp/nccp/en. Accessed March $22,2017$. 